\title{
Chronic Granulomatous Disease Due to a Defect in the Cytosolic Factor Required for Nicotinamide Adenine Dinucleotide Phosphate Oxidase Activation
}

\author{
John T. Curnutte, Roger L. Berkow, Robert L. Roberts, Susan B. Shurin, and Patricia J. Scott \\ Department of Basic and Clinical Research, Research Institute of Scripps Clinic, La Jolla, California 92037; Department of Pediatrics, \\ University of Alabama at Birmingham, Birmingham, Alabama 35233; Department of Pediatrics, UCLA School of Medicine, Los \\ Angeles, California 90024; Department of Pediatrics, Case Western Reserve University School of Medicine, Cleveland, Ohio 44106
}

\begin{abstract}
The superoxide-generating enzyme of human neutrophils, NADPH oxidase, is present in a dormant state in unstimulated neutrophils. It can be converted to an active form in a cell-free system if both the plasma membrane and cytosol fractions are incubated together in the presence of arachidonic acid. This system was used to determine the nature of the biochemical defect in seven patients with the autosomal recessive, cytochrome $b$-positive form of chronic granulomatous disease (CGD). A severe deficiency in the cytosol factor was identified in each patient. The defective activity was not caused by the presence of an inhibitor, nor could it be restored to normal by combining cytosol fractions from different patients. In contrast, the membrane fractions from all seven patients contained normal levels of NADPH oxidase when activated in the presence of control cytosol. Of family members tested (obligate heterozygotes for this disorder), seven of eight had intermediate levels of cytosol factor activity. The respiratory burst defect in this form of CGD is caused by an abnormality in the cytosolic factor required for NADPH oxidase activation.
\end{abstract}

\section{Introduction}

Chronic granulomatous disease (CGD) ${ }^{1}$ is a congenital disorder in which phagocytic cells are unable to generate hydrogen peroxide, superoxide $\left(\mathrm{O}_{2}^{-}\right)$, and various other oxygen radicals in response to microorganisms (1). Since these agents play

Address correspondence to Dr. John Curnutte, Department of Basic and Clinical Research, BCR-7, Scripps Clinic and Research Foundation, 10666 North Torrey Pines Road, La Jolla, CA 92037.

A portion of this work was presented in abstract form at the American Society of Hematology National Meeting, New Orleans, LA, December 1985, and at the American Federation for Clinical Research National Meeting, Washington, DC, May, 1986 (1985. Blood. 66:77a; 1986. Clin. Res. 34:455a).

Received for publication 25 June 1987 and in revised form 22 September 1987.

1. Abbreviations used in this paper: CGD, chronic granulomatous disease; $\mathrm{O}_{2}^{-}$, superoxide; $\mathrm{NBT}$, nitroblue tetrazolium; PMA, phorbol 12myristate 13-acetate.

J. Clin. Invest.

(C) The American Society for Clinical Investigation, Inc.

$0021-9738 / 88 / 02 / 0606 / 05 \quad \$ 2.00$

Volume 81, February 1988, 606-610 a critical role in the microbicidal systems of the phagocyte, patients with CGD suffer from recurrent infections. It has long been recognized that the phenotype of CGD can be caused by at least two genetic mutations, one X-linked and the other autosomal recessive (1). More recently, these two forms of the disease have each been subclassified based on the presence or absence of cytochrome $b_{558}$, a heme protein believed to be a component of the superoxide-generating enzyme, NADPH oxidase (2-4). That at least three of these four possible forms of CGD are genetically distinct has been demonstrated by complementation studies using hybridized monocytes derived from patients with different forms of $\operatorname{CGD}(4,5)$.

Numerous studies have demonstrated that homogenates of CGD cells do not contain measurable NADPH oxidase activity $(6,7)$. The failure to observe oxidase activity can be explained by either a defect in the enzyme itself or an abnormality in the pathway responsible for its activation. Until recently, however, it has not been possible to distinguish clearly between these two possibilities. The development of a cell-free system for activating dormant NADPH oxidase from unstimulated neutrophils has provided new tools for the study of this question (8-11).

In the cell-free system, NADPH oxidase from disrupted unstimulated neutrophils can be activated by AA provided that both the cytosol and membrane-containing fractions are present. We have previously shown that the factor present in the cytosol was normal in eight patients with either the $\mathrm{X}$ linked or autosomal recessive, cytochrome $b$-negative forms of $\operatorname{CGD}(9,12)$. In contrast, the membrane fraction from each of these patients failed to generate $\mathrm{O}_{2}^{-}$in the presence of normal cytosol. The observation that the cytosol factor is absolutely required for NADPH oxidase activation in the cell-free system raised the possibility that a defect in this factor might be responsible for one of the forms of CGD. In this report we describe seven patients with the autosomal recessive, cytochrome $b$-positive form of CGD whose disease appears to be due to a severe deficiency of cytosol factor activity. The plasma membrane fraction in each patient, on the other hand, could be activated to generate a normal rate of $\mathrm{O}_{2}^{-}$in the presence of control cytosol. In addition, we have been able to identify the carrier state in this autosomal recessive form of CGD on the basis of intermediate levels of cytosol factor in all but one of the parents tested from these families.

\section{Methods}

Chemicals. AA was obtained in the free acid form from Nu-Chek Prep. Inc., Elysian, MN. SOD from bovine erythrocytes, ferricytochrome $c$ 
(Type VI), NADPH (Type I), nitroblue tetrazolium (NBT), and phorbol 12-myristate 13-acetate (PMA), were obtained from Sigma Chemical Co., St. Louis, MO. Macrodex (dextran 70), Ficoll-Paque, and Percoll were products of Pharmacia, Inc., Piscataway, NJ. Deionized distilled water was used throughout all experiments and was obtained using either an ultra-pure water filter (Barnstead Co., Sybron Corp., Boston, MA) or a Milli-Q system (Millipore Continental Water Systems, Bedford, MA). All other chemicals used were of at least reagent grade.

Preparation of neutrophils. Human neutrophils of $\geq 98 \%$ purity were prepared as previously described (12) using acid citrate dextrose as the anticoagulant, dextran to sediment erythrocytes, and FicollPaque to separate mononuclear cells from neutrophils.

Patients with CGD. We studied four female patients and three male patients with autosomal recessive CGD. All seven patients were found to have normal levels of cytochrome $b$ (Table II). Four of the patients (see Table II for patient numbers) have been previously described in the literature: patient 2(13), patient 3 (14), patient 5 (15), and patient 7 (16). Patient 5 was also reported to have an NADPH oxidase with a diminished affinity for its substrate (15).

Disruption of cells and fractionation on Percoll gradients. Neutrophils were disrupted by nitrogen cavitation and the postnuclear supernatant was fractionated on discontinuous Percoll gradients by a modification of the method of Borregaard et al. (17) as previously described (12). After the removal of Percoll, all particulate fractions were resuspended at a concentration of $12.5 \times 10^{8}$ cell equivalents $/ \mathrm{ml}(4.0 \pm 0.9$ SD $\mathrm{mg}$ protein $/ \mathrm{ml}$ ) as previously described (18) and stored at $-70^{\circ} \mathrm{C}$ for no longer than $2 \mathrm{~d}$ before being assayed. The $230,000-\mathrm{g}$ supernatant of the cytosol fraction was likewise stored at $-70^{\circ} \mathrm{C}$ at a concentration of $2-7.5 \times 10^{7}$ cell equivalents $/ \mathrm{ml}\left(250 \pm 16 \mathrm{SD} \mu \mathrm{g}\right.$ protein $/ 10^{7}$ cell equivalents).

NADPH oxidase. The level of NADPH oxidase activity before and after stimulation with AA in the cell-free activation system was determined as previously described using method $B$, in which NADPH was used to initiate $\mathrm{O}_{2}^{-}$generation after a 3-min preincubation of all the other reaction constituents (including AA) (12). Superoxide-dependent cytochrome $c$ reduction was monitored continuously and the maximal velocity of the reaction was used to calculate oxidase activity.

Determination of cytosol factor levels in CGD carriers. Measurements of intermediate levels of cytosol factor in various members of the patients' families were performed using the standard activation method described above at concentrations of cytosol ranging between 0.2 and $1.0 \times 10^{7}$ cell equivalents/reaction (total volume $0.75 \mathrm{ml}$ ). The extent to which these various concentrations of cytosol were able to activate control membranes was then compared to the rates obtained with control cytosol which had been prepared and assayed concurrently with the family samples. The results for both were plotted both in terms of cell equivalents of cytosol as well as per mg of cytosol protein. The concentration of cytosol factor was then expressed as the percent of control by dividing the quantity of control cytosol by the quantity of the test cytosol which gave comparable rates $\mathrm{O}_{2}^{-}$production.

Intact cell superoxide generation and NBT reduction. Intact cell superoxide production was measured as previously described using PMA and AA as stimuli (19). NBT reduction was determined using the same reaction mixture except NBT $(1 \mathrm{mg} / \mathrm{ml})$ was substituted for cytochrome $c$. After $5 \mathrm{~min}$ of stimulation, the NBT reaction mixture was placed on ice and the percentage of positive cells was determined microscopically as previously described (20).

\section{Results}

Table I shows the results of two experiments in which membranes and cytosol from CGD patients were mixed with those from controls in different combinations. When CGD cytosol was mixed with CGD membranes, negligible rates of $\mathrm{O}_{2}^{-}$generation were detected $(<1 \%$ of control). The defect in oxidase
Table I. Cell-free Activation of NADPH Oxidase in Autosomal Recessive, Cytochrome b-positive CGD Neutrophils

\begin{tabular}{|c|c|c|c|}
\hline \multicolumn{2}{|c|}{ Source of fraction } & \multicolumn{2}{|c|}{$\mathrm{O}_{2}^{-}$generation } \\
\hline \multirow[t]{2}{*}{ Cytosol factor } & Membranes & Patient 1 & Patient 7 \\
\hline & & \multicolumn{2}{|c|}{$\begin{array}{l}\text { nmol/min } / 10^{7} \text { cell eq of } \\
\text { membrane }\end{array}$} \\
\hline Control & Control & 29.7 & 58.5 \\
\hline CGD & CGD & 0.1 & 0.3 \\
\hline Control & CGD & 29.8 & 50.1 \\
\hline CGD & Control & 0.7 & 4.1 \\
\hline $\begin{array}{l}50 \% \text { CGD/ } \\
50 \% \text { Control }\end{array}$ & Control & 15.0 & 24.4 \\
\hline
\end{tabular}

Superoxide generation was measured as described in Methods, with each reaction mixture containing $7 \times 10^{6}$ cell equivalents of membranes, $10^{7}$ cell equivalents of cytosol, and $0.082 \mathrm{mM} \mathrm{AA}$ as the activating agent. The results of two experiments are shown, each performed with a different patient and control. Each patient was studied once and the data shown are the means of measurements performed in duplicate.

activation was not due to an abnormality in the membrane fraction, as the CGD membranes were capable of generating normal rates of superoxide when activated in the presence of control cytosol. Instead, there was a marked abnormality in cytosol factor activity. Control membranes activated in the presence of cytosol from each of the patients produced $\mathrm{O}_{2}^{-}$at rates that were 2.4 and $7 \%$ of control. (The observation that the cytosol defect was even more striking [ 0.3 and $0.6 \%$ of control] when each patient's cytosol was tested against his own membranes appears to be due to small amounts of cytosol factor which remain associated with some preparations of control membranes.) The defect in the CGD cytosol did not appear to be caused by an inhibitor, as evidenced by the failure of CGD cytosol to inhibit control cytosol factor activity when mixed together. The negligible oxidase activity in the presence of CGD cytosol also did not appear to be due to an altered affinity for NADPH caused by the CGD cytosol. In control experiments not shown using cytosol from patients 1,5 , and 7 , NADPH concentrations as high as $1 \mathrm{mM}$ failed to improve the rate of $\mathrm{O}_{2}^{-}$generation by either patient or control membranes. This control was of particular importance in the studies on patient 5 , as she has been previously reported (15) to have a kinetic defect in her NADPH oxidase manifested by a fourfold decrease in the affinity of the enzyme for NADPH. When activated in the presence of normal cytosol, this patient's membrane oxidase had a normal $K_{\mathrm{m}}$ for NADPH (41 $\mu \mathrm{M}$ vs. $36 \mu \mathrm{M}$ for control). Due to insufficient patient cytosol, it was not possible to determine the $K_{\mathrm{m}}$ of her membrane oxidase when activated in the presence of her own cytosol.

Table II summarizes the respiratory burst studies performed on all seven patients with the autosomal recessive, cytochrome $b$-positive form of CGD. Four females and three males were identified as having this form of the disease based upon pedigree analysis, failure to detect a carrier state in the mothers on an NBT test, and the presence of normal levels of cytochrome $b$ in the patients' neutrophils. In six of the seven patients, neutrophils failed to reduce NBT when stimulated with PMA. In patient $5,85 \%$ of the cells did stain with NBT, although the intensity of the stain was weak. Interestingly, this 
Table II. Levels of Respiratory Burst Components in Autosomal Recessive, Cytochrome b-positive CGD

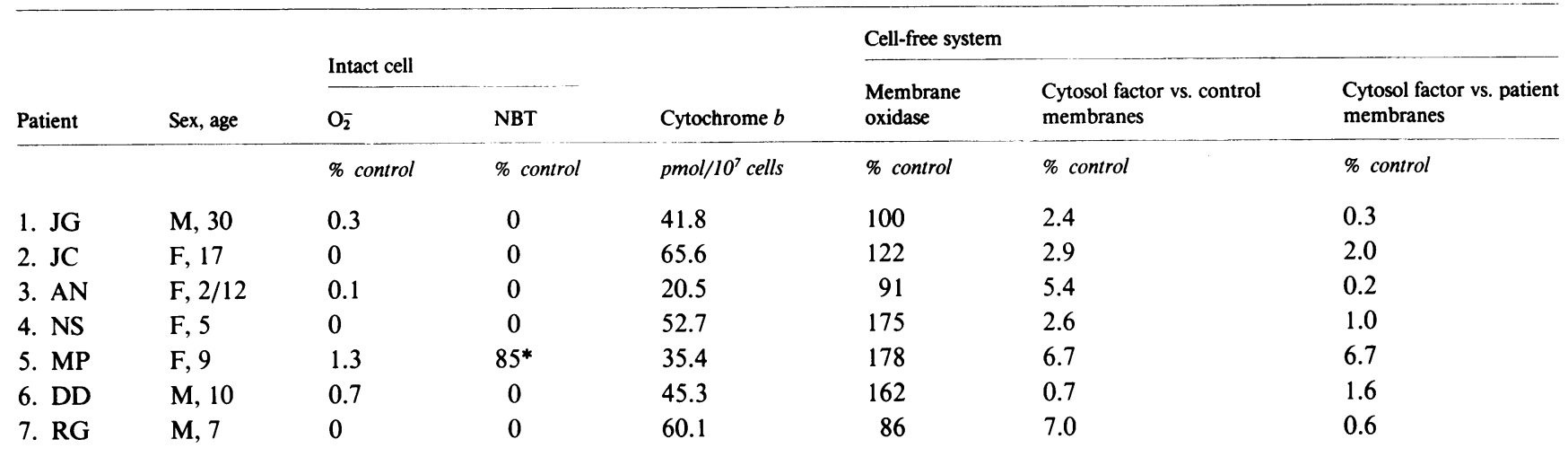

Intact cell $\mathrm{O}_{2}^{-}$generation and NBT reduction were measured in the presence of $3.25 \mu \mathrm{M}$ PMA as described in Methods and expressed the percent of control cell values obtained the same day (control $\mathrm{O}_{2}^{-}$rates: $95.2 \pm 21.9 \mathrm{SD} \mathrm{nmol} / \mathrm{min} \cdot 10^{7}$ cells $(n=7$ ); control NBT reduction: $\geq 90 \%$ positive cells). Cytochrome $b$ levels were measured on the postnuclear supernatant from nitrogen-cavitated neutrophils as described in Methods, with control cells containing $43.3 \pm 10.5 \mathrm{SD}$ pmol cytochrome $b / 10^{7}$ cells $(n=11)$. Cell-free activation of NADPH oxidase was performed as outlined in Methods and Table I. The level of the arachidonate-activatable membrane component of NADPH oxidase was determined by measuring the rate of $\mathrm{O}_{2}^{-}$generated by either control or patient membranes $\left(6-12.5 \times 10^{6}\right.$ cell equivalents/reaction) in the presence of control cytosol ( $10^{7}$ cell equivalents/reaction). Each patient level is expressed as the percent of the control value obtained the same day. Cytosol factor levels were similarly measured by comparing the maximal rate of $\mathrm{O}_{2}^{-}$generated in the presence of patient cytosol (10 cell equivalents/reaction) to that obtained with control cytosol prepared the same day. Cytosol factor levels were measured using either control or patient membranes (6-12.5 $\times 10^{6}$ cell equivalents/reaction) and the results from both types of assays are shown (see text for explanation). Control levels for oxidase activation ranged between 4.8 and $58.8 \mathrm{nmol} \mathrm{O}_{2}^{-} / \mathrm{min} \cdot 10^{7}$ cell equivalents of membranes in the presence of $10^{7}$ cell equivalents of cytosol. The higher levels of oxidase activation were obtained in later experiments when membranes were harvested at greater concentrations, which improved the stability of the oxidase. Patients 1-3 were each studied twice with a representative experiment shown for each while the remaining patients were studied once. The data shown are the means of duplicate determinations made within a single experiment. ${ }^{*}$ All cells were only weakly stained.

patient had the highest level of cytosol factor activity $(6.7 \%$ of control) when tested against both her own and control membranes. The rate of $\mathrm{O}_{2}^{-}$production by each patient's intact cells in response to PMA was also examined. Four of the seven patients were found to generate trace amounts of $\mathrm{O}_{2}^{-}$, again with the highest rate ( $1.3 \%$ of control) seen in patient 5 . As shown in Table II, cytosol factor levels ranged between 0.7 and $7.0 \%$ of control in this series of patients when assayed against control membranes. When tested against CGD membranes, cytosol factor levels were $\leq 2.0 \%$ in six of the patients. In contrast, the level of arachidonate-activatable NADPH oxidase in the membrane fractions from all of the patients was at or above control levels.

Since this form of CGD is inherited in an autosomal recessive manner, it was of interest to determine whether intermediate levels of cytosol factor were present in the neutrophils of obligate heterozygotes. Table III summarizes the findings from these studies. In four of the five mothers tested and in both fathers examined using the cell-free system, intermediate levels of cytosol factor activity were found when calculated on either a per-cell or per-milligram basis. The sister of patient 1 also had an intermediate cytosol factor level. The average level for all eight family members was $55 \%$ of control when expressed in terms of cell equivalents of cytosol. In contrast to this clear demonstration of the carrier state in the majority of family members were the results obtained with intact cell measurements of respiratory burst activity. In these studies, most of the family members had normal levels of both $\mathrm{O}_{2}^{-}$production and NBT reduction in response to PMA. These results suggest that the cytosol factor concentration in normal neutrophils is saturating with respect to oxidase activation, and that in most individuals, an $\sim 50 \%$ decrease in cytosol factor levels does not affect the magnitude of respiratory burst.

Since the defect in this group of patients involves the cytosol fraction, it is possible to perform mixing experiments using cytosol fractions from different patients to test whether there is any detectable heterogeneity within the group. Mixing of cytosols from patients 1 and 3,2 and 4, or 1 and 7 failed to restore normal cytosol factor activity. The small quantities of cytosol obtained from the other patients precluded the testing of other combinations. The results of these mixing studies suggest that there is a common defect in the cytosol in this group of autosomal recessive, cytochrome $b$-positive patients.

\section{Discussion}

The severe respiratory burst defect in autosomal recessive, cytochrome $b$-positive CGD can be explained on the basis of the findings presented above. In previous studies, we $(9,12)$ and others $(8,10,11,21-23)$ have demonstrated that the activation of dormant NADPH oxidase in the cell-free system requires the presence of a cytosol factor. Without it, oxidase cannot be activated in these systems. The finding that patients with this form of CGD are severely deficient in this cytosol factor can therefore account for their respiratory burst defect. Further support that this deficiency is the underlying basis for this type of CGD is provided by the family studies in Table III, which demonstrate the expected intermediate levels of cytosol factor in seven of the eight family members tested. These studies also indicate that the cytosol factor is not merely an artifact of the cell-free system, but is essential for oxidase activation in the intact cell. 
Table III. Demonstration of the Carrier State in Autosomal Recessive, Cytochrome b-positive CGD Families

\begin{tabular}{|c|c|c|c|c|}
\hline \multirow[b]{3}{*}{ Family } & \multirow{2}{*}{\multicolumn{2}{|c|}{ Intact cells }} & \multicolumn{2}{|c|}{ Cytosol factor level } \\
\hline & & & \multirow{2}{*}{$\begin{array}{l}\text { per cell } \\
\text { equivalents }\end{array}$} & \multirow{2}{*}{$\begin{array}{l}\text { per } \mathrm{mg} \\
\text { protein }\end{array}$} \\
\hline & $\mathrm{O}_{2}^{-}$ & NBT & & \\
\hline & $\%$ control & $\%$ control & $\%$ control & $\%$ contro \\
\hline 1. Mother & 114 & 83 & 97 & 109 \\
\hline Father & 77 & 74 & 53 & 73 \\
\hline Sister & 72 & 78 & 53 & 67 \\
\hline 3. Mother & 61 & 75 & 62 & 61 \\
\hline Father & 100 & ND & 46 & 46 \\
\hline 4. Mother & 120 & 98 & 42 & 57 \\
\hline 6. Mother & 140 & 100 & 46 & 56 \\
\hline 7. Mother & 131 & 97 & 42 & 52 \\
\hline Mean & $102 \pm 10 \mathrm{SE}$ & $86 \pm 4$ & $55 \pm 6$ & $65 \pm 7$ \\
\hline
\end{tabular}

Intact cell $\mathrm{O}_{2}^{-}$generation and NBT reduction were measured as described in Table II using PMA ( $3.25 \mu \mathrm{M})$ as the stimulus. Cytosol factor levels were also measured as described in Table II and are expressed as the percent of control values determined concurrently as outlined in Methods. Each value represents the mean of duplicate measurements performed at four different concentrations of cytosol (range $0-1.0 \times 10^{7}$ cell equivalents/reaction). Each family member was studied once, except for the mother of patient 3 , who was studied twice (a representative experiment is shown). The family number corresponds to the patient number cited in Table II.

The identity of the cytosol factor has not yet been established. Previous studies have shown that it exists in at least two forms based on apparent molecular weight from gel filtration chromatography $(12,22)$. We have reported that the factor appears to be a $240-\mathrm{kD}$ protein that may dissociate under some circumstances into $\sim 40-\mathrm{kD}$ subunits (12). The cytosol factor can be resolved from protein kinase $\mathrm{C}$ under these chromatographic conditions and does not require calcium or ATP for activity. The cytosol factor does require $\mathrm{Mg}^{2+}$ for activity, but beyond this no other low molecular weight substances have been found to be required. Attempts to further purify the cytosol factor in our laboratory have been frustrated by the instability of the partially purified material.

The autosomal recessive, cytochrome $b$-positive form of CGD can be readily distinguished from both the X-linked, cytochrome $b$-negative and autosomal recessive, cytochrome $b$-negative forms of CGD in the cell-free system. In the latter two forms of CGD, the cytosol factor is normal while the membranes are defective $(9,12)$. The opposite is the case in autosomal recessive, cytochrome $b$-positive CGD. Of interest is the correlation between the presence or absence of cytochrome $b$ and whether the dormant oxidase in the membrane fraction can be activated by arachidonate. In both of the genetic forms of cytochrome $b$-negative CGD, the membranes are defective, while in the cytochrome $b$-positive form discussed in this report, the membranes can be normally activated with control cytosol. There is now little doubt that cytochrome $b$ plays an important role in NADPH oxidase function, although its precise biochemical role in oxidase function has yet to be determined. The recent cloning of the gene defective in X-linked CGD (24) and the finding that this gene encodes the $91-\mathrm{kD}$ subunit of the cytochrome $b$ heterodimer
(25) have unequivocally established that the absence of cytochrome $b$ leads to a defect in the respiratory burst.

The clinical severity of autosomal recessive, cytochrome $b$-positive CGD appears to be less than that seen in the cytochrome $b$-negative forms, an observation also made by Tauber et al. in their review of CGD (26). In the present series, CGD was not diagnosed in patients 1 and 6 until the ages of 30 and 9, respectively, when each developed hepatic abscesses. Their previous medical histories were significant only for impetiginous skin lesions. Patient 2 has had only one serious illness, Legionella pneumonia, in addition to occasional skin infections (13). Patient 5 (who has the highest level of cytosol factor activity) has been healthy and was diagnosed only after her male sibling died of Aspergillus pneumonitis (15). Our findings that these patients have low, but detectable, levels of cytosol factor activity and that the membrane component of NADPH oxidase is normal suggest that the small amount of $\mathrm{O}_{2}^{-}$generated by their neutrophils may, when amplified by the myeloperoxidase-dependent bactericidal system, be sufficient to protect against some types of infections.

\section{Acknowledgments}

We thank Susan Burke and Carol Fedoryszyn for their excellent help in the preparation of this manuscript. We also thank Drs. Bernard Huss, Stanley Schwartz, Myron Liebhaber, and William Tisdale for kindly allowing us to obtain blood samples from their patients.

This work was supported in part by U. S. Public Health Service grants AI-24838, AI-22048, HD-08900, and RR-00833, and by grant R0066-01 from the Cystic Fibrosis Foundation. Dr. Curnutte is an Established Investigator of the American Heart Association.

\section{References}

1. Curnutte, J. T., and B. M. Babior. 1987. Chronic granulomatous disease. Adv. Hum. Genet. 16:229-297.

2. Segal, A. W., A. R. Cross, R. C. Garcia, N. Borregaard, N. Valerius, J. F. Soothill, and O. T. G. Jones. 1983. Absence of cytochrome b-245 in chronic granulomatous disease: a multicenter European evaluation of its incidence and relevance. $N$. Engl. J. Med. 308:245-251.

3. Bohler, M.-C., R. A. Seger, R. Mouy, E. Vilmer, A. Fischer, and C. Griscelli. 1986. A study of 25 patients with chronic granulomatous disease: a new classification by correlating respiratory burst, cytochrome b, and flavoprotein. J. Clin. Immunol. 6:136-145.

4. Weening, R. S., L. Corbeel, M. de Boer, R. Lutter, R. van Zwieten, M. N. Hamers, and D. Roos. 1985. Cytochrome b deficiency in an autosomal form of chronic granulomatous disease: a third form of chronic granulomatous disease recognized by monocyte hybridization. J. Clin. Invest. 75:915-920.

5. Hamers, M. N., M. de Boer, L. J. Meerhof, R. S. Weening, and D. Roos. 1984. Complementation in monocyte hybrids revealing genetic heterogeneity in chronic granulomatous disease. Nature (Lond.). 307:553-555.

6. Hohn, D. C., and R. I. Lehrer. 1975. NADPH oxidase deficiency in X-linked chronic granulomatous disease. J. Clin. Invest. 55:707713.

7. Curnutte, J. T., R. S. Kipnes, and B. M. Babior. 1975. Defect in pyridine nucleotide dependent superoxide production by a particulate fraction from the granulocytes of patients with chronic granulomatous disease. N. Engl. J. Med. 293:628-632.

8. Bromberg, Y., and E. Pick. 1985. Activation of NADPH-dependent superoxide production in a cell-free system by sodium dodecyl sulfate. J. Biol. Chem. 260:13539-13545.

9. Curnutte, J. T. 1985. Activation of human neutrophil nicotinamide adenine dinucleotide phosphate, reduced (triphosphopyridine 
nucleotide, reduced) oxidase by arachidonic acid in a cell-free system. J. Clin. Invest. 75:1740-1743.

10. Heyneman, R. A., and R. E. Vercauteren. 1984. Activation of a NADPH oxidase from horse polymorphonuclear leukocytes in a cellfree system. J. Leukocyte Biol. 36:751-759.

11. McPhail, L. C., P. S. Shirley, C. C. Clayton, and R. Snyderman. 1985. Activation of the respiratory burst enzyme from human neutrophils in a cell-free system: evidence for a soluble cofactor. J. Clin. Invest. 75:1735-1739.

12. Curnutte, J. T., R. Kuver, and P. J. Scott. 1987. Activation of neutrophil NADPH oxidase in a cell-free system. Partial purification of components and characterization of the activation process. J. Biol. Chem. 262:5563-5569.

13. Peerless, A. G., M. Liebhaber, S. Anderson, R. I. Lehrer, and E. R. Stiehm. 1985. Legionella pneumonia in chronic granulomatous disease. J. Pediatr. 106:783-785.

14. Schoumacher, R. A., R. L. Berkow, and R. E. Tiller. 1987. Invasive pulmonary aspergillosis in an infant: an unusual presentation of chronic granulomatous disease. Pediatr. Infect. Dis. 6:215-216.

15. Shurin, S. B., H. J. Cohen, J. C. Whitin, and P. E. Newburger. 1983. Impaired granulocyte superoxide production and prolongation of the respiratory burst due to a low-affinity NADPH-dependent oxidase. Blood. 62:564-571.

16. Ohno, Y., E. S. Buescher, R. Roberts, J. A. Metcalf, and J. I. Gallin. 1986. Reevaluation of cytochrome $b$ and flavin adenine dinucleotide in neutrophils from patients with chronic granulomatous disease and description of a family with probable autosomal recessive inheritance of cytochrome b deficiency. Blood. 67:1132-1138.

17. Borregaard, N., J. M. Heiple, E. R. Simon, and R. A. Clark. 1983. Subcellular localization of the b-cytochrome component of the human neutrophil microbicidal oxidase: translocation during activation. J. Cell Biol. 97:52-61.

18. Curnutte, J. T., R. Kuver, and B. M. Babior. 1987. Activation of the respiratory burst oxidase in a fully soluble system from human neutrophils. J. Biol. Chem. 262:6450-6452.

19. Badwey, J. A., J. T. Curnutte, J. M. Robinson, C. B. Berde, M. J. Karnovsky, and M. L. Karnovsky. 1984. Effects of free fatty acids on release of superoxide and on change of shape by human neutrophils: reversibility by albumin. J. Biol. Chem. 259:7870-7877.

20. Baehner, R. L., and D. G. Nathan. 1968. Quantitative nitroblue tetrazolium test in chronic granulomatous disease. N. Engl. J. Med. 278:971-976.

21. Cox, J. A., A. Y. Jeng, N. A. Sharkey, P. M. Blumberg, and A. I. Tauber. 1985. Activation of the human neutrophil nicotinamide adenine dinucleotide phosphate (NADPH)-oxidase by protein kinase C. $J$. Clin. Invest. 76:1932-1938.

22. Gabig, T. G., D. English, L. P. Akard, and M. J. Schell. 1987. Regulation of neutrophil NADPH oxidase activation in a cell-free system by guanine nucleotides and fluoride: evidence for participation of a pertussis and cholera toxin-insensitive G protein. J. Biol. Chem. 262:1685-1690.

23. Clark, R. A., K. G. Leidal, D. W. Pearson, and W. M. Nauseef. 1987. NADPH oxidase of human neutrophils: subcellular localization and characterization of an arachidonate-activatable superoxide-generating system. J. Biol. Chem. 262:4065-4074.

24. Royer-Pokora, B., L. M. Kunkel, A. P. Monaco, S. C. Goff, P. E. Newburger, R. L. Baehner, F. S. Cole, J. T. Curnutte, and S. H. Orkin. 1986. Cloning the gene for an inherited human disorderchronic granulomatous disease-on the basis of its chromosomal location. Nature (Lond.). 322:32-38.

25. Dinauer, M. C., S. H. Orkin, R. Brown, A. J. Jesaitis, and C. A. Parkos. 1987. The glycoprotein encoded by the X-linked chronic granulomatous disease locus is a component of the neutrophil cytochrome b complex. Nature (Lond.). 327:717-720.

26. Tauber, A. I., N. Borregaard, E. Simons, and J. Wright. 1983. Chronic granulomatous disease: a syndrome of phagocyte oxidase deficiencies. Medicine (Baltimore). 62:286-309. 